\title{
BRAVE NEW WORLDS IN STABLE HOMOTOPY THEORY
}

\author{
J. P. MAY
}

I will give a broad survey of the general area of structured ring spectra in modern constructions of the stable homotopy category. I will give some background and history, but my main focus will be a description of work in progress of Mike Mandell, Stefan Schwede, Brooke Shipley, and myself $[24,25]$. By "modern" I mean that there must be a smash product that gives a point-set level symmetric monoidal structure on a closed model category. The associated homotopy category that is obtained by inverting the weak equivalences must be equivalent to the classical stable homotopy category, or at least to a reasonably large full subcategory.

We define a strict ring spectrum or strict commutative ring spectrum to be a monoid or commutative monoid in any such symmetric monoidal category of spectra. In a really good category, the categories of monoids and of commutative monoids should themselves be model categories. All model categories in sight should be tensored and cotensored over either the category of spaces or the category of simplicial sets. I shall work throughout with spaces for definiteness. I shall focus on strict commutative ring spectra since their study is much deeper and more interesting than the study of merely associative strict ring spectra.

I will start with a little history. Spectra were first introduced by Lima [21], a student of Spanier at Chicago, in 1958. There was quite a bit of work by many people, including Adams and Puppe, trying to construct a good stable homotopy category before the problem was solved by Mike Boardman in his $1964 \mathrm{PhD}$ thesis at Warwick [4], which is still unpublished. A popularized, and in some respects bowdlerized, version of Boardman's category was explained and written up by Adams in lectures given at Chicago in 1971 [1]. A parallel development of a category of simplicial spectra was begun by Kan in 1963 [16] although the crucial point, the definition of smash products, was only addressed later, in work of Kan and Whitehead [17] that was completed by Burghelea and Deleanu [7].

In the work that I have described so far, and in many later alternative constructions, the smash product only became associative, commutative, and unital after passage to the stable homotopy category. For that reason, ring and module spectra have traditionally been flabby homotopy theoretic objects, very useful for many applications, but hopelessly inadequate for others. In such a context, the cofiber of a map of module spectra is not in general again a module spectrum, hence one cannot do serious homotopy theory in categories of modules over ring spectra. There are many other such defects.

I began looking for a way of constructing the stable homotopy category that would have good point-set level properties almost 30 years ago. In particular, I advertised the idea that a spectrum should be a sequence of spaces $E_{i}$ together with homeomorphisms $E_{i} \cong \Omega E_{i+1}$ at a conference in 1968 [26]. In 1972, Frank

1991 Mathematics Subject Classification. 55P42.

The author was supported in part by NSF Grant \#DMS-9423300. 
Quinn, Nigel Ray and I introduced $E_{\infty}$-ring spectra [27], and we showed that Thom spectra gave naturally occurring examples.

The work with Quinn and Ray led me to develop a multiplicative elaboration of infinite loop space theory $[27,29]$ that allowed passage back and forth between $E_{\infty}$ ring spaces and $E_{\infty}$-ring spectra. This allowed the construction of $E_{\infty}$-ring spectra from bipermutative categories, such as categories of modules over commutative rings with their direct sums and tensor products. This gave a plentiful supply of $E_{\infty}$-ring spectra, such as the Eilenberg-Mac Lane spectra of commutative rings, the algebraic $K$-theory spectra of commutative rings and the connective topological $K$-theory spectra.

The passage back and forth between $E_{\infty}$-ring spaces and $E_{\infty}$-ring spectra was vital to calculations made in the 1970's, for example the calculation of characteristic classes for topological bundles and the calculation of the cobordism groups of topological manifolds. One point is that the operad actions in terms of which these structures were defined gave rise to computable homology operations. Another is that the point set level structures allowed the construction and descriptive analysis of new infinite loop spaces with computable invariants. For example, work of Sullivan [37] and many others led to a proof that, at an odd prime $p, B S T o p$ is equivalent as an infinite loop space to the classifying space of $k O$-oriented spherical fibrations. Work of Quillen [32] and others led to a discrete model description of $k O$ as an algebraic $K$-theory type spectrum associated to finite fields. These ideas allowed me to reduce the calculation of characteristic classes for topological bundles at odd primes to calculations in the cohomology of finite groups [8, 28].

Paul Goerss and Mike Hopkins [12] have recently developed another, obstruction theoretic, way of constructing $E_{\infty}$-ring spectra. Here again, the operad actions that go into the description of $E_{\infty}$-ring spectra are critical to both the theory and the calculations that make the constructions work.

During the work with Quinn and Ray, it became apparent that spectra should be defined in a coordinate-free fashion, with spaces $E V$ indexed on finite dimensional inner product spaces $V$ contained in a given universe $U$ isomorphic to $\mathbb{R}^{\infty}$. There should be homeomorphisms $E V \cong \Omega^{W-V} E W$ whenever $V \subset W$, where $W-V$ is the orthogonal complement of $V$ in $W$.

Thinking equivariantly, tom Dieck [10] had realized the advantages of such indexing even earlier, the $V$ 's being representations contained in a $G$-universe $U$ for some compact Lie group $G$. Lewis and I [20] worked out details of the coordinate-free approach to the stable homotopy category in the early 1980's. Our focus was on the equivariant stable homotopy category, and our work gave the first and, so far, only treatment of the equivariant foundations of stable homotopy theory. A more readable account appears in "Equivariant homotopy and cohomology theory" [30].

Building on both the work with Quinn and Ray and the work with Lewis, a complete solution to the problem of constructing a symmetric monoidal category of spectra was finally obtained by Elmendorf, Kriz, Mandell, and myself in "Rings, modules, and algebras in stable homotopy theory" [11]; I will refer to that book as EKMM. The theory works in full equivariant generality, and it ties in well with all of the previous work in the area. We gave a variety of applications, and more has been done since the book was written. We call our category the category of $S$-modules, denoted $\mathscr{M}_{S}$, where $S$ is the sphere spectrum. These are coordinate free 
spectra with additional structure. The additional structure allows the construction of a symmetric monoidal smash product.

A commutative $S$-algebra $R$ is a commutative monoid in $\mathscr{M}_{S}$ : it has a commutative, associative, and unital product $R \wedge_{S} R \longrightarrow R$ with unit $S \longrightarrow R$. $R$-modules are defined similarly in terms of maps $R \wedge_{S} M \longrightarrow M$. The category $\mathscr{M}_{R}$ of $R$ modules has all of the good properties of $\mathscr{M}_{S}$. The derived homotopy category $\mathscr{D}_{R}$ is obtained by inverting the weak equivalences, which are the maps of $R$-modules that are weak equivalences of spectra. For example, the derived category $\mathscr{D}_{H k}$ associated to the Eilenberg-Mac Lane $S$-algebra $H k$ is equivalent to the classical derived category of chain complexes of $k$-modules, and the equivalence converts smash products and function $H k$-modules to tensor product and hom functors. The derived categories of modules over real and complex, periodic and connective, K-theory have been analyzed algebraically by Jerome Wolbert [39].

Working in the derived category of $M U$-modules, we gave quick and easy constructions as $M U$-modules of spectra $(M U / I)\left[Y^{-1}\right]$, including Brown-Peterson, Johnson-Wilson, and Morava $K$-theory spectra. At least at odd primes, these are all easily shown to be commutative $M U$-ring spectra in the homotopical sense and therefore commutative ring spectra in the classical sense. Neil Strickland [36] has extended the results of EKMM to the 2-local case, proving for example that $B P$ (at 2 ) is a commutative $M U_{(2)}$-ring spectrum. Similar arguments will apply starting with $B P$ once Kriz's sketch proof [18] that $B P$ is an $E_{\infty}$-ring spectrum is completed. Maria Basterra [3] has worked out a rigorous construction of André Quillen cohomology for commutative $S$-algebras, which is the essential tool. In some combination of authorship, I expect Basterra, Kriz, Hunter, and McClure to complete this work.

A deep fact is that the category of commutative $R$-algebras is itself a model category, with good control on the homotopical behavior of the underlying $R$-modules. For any $R$-module $E$, the Bousfield localization $A_{E}$ of an $R$-algebra $A$ is an $R$ algebra. For example, $K U=k u_{K U}$ and $K O=k o_{K O}$, giving a quick proof that $K U$ is a $k u$-algebra and therefore an $S$-algebra, and similarly for $K O$.

When $R=H k$, Mike Mandell [23] has proven that the derived homotopy category of commutative $H k$-algebras is equivalent to the algebraic derived category of $E_{\infty}-k$-algebras. He has used this together with an unpublished theorem of Dwyer and Hopkins to prove that the singular cochain functor with coefficients in the algebraic closure of the field with $p$ elements gives a contravariant equivalence from the homotopy category of $p$-nilpotent spaces of finite type to a full subcategory of the category of $E_{\infty}$-algebras over $\bar{F}_{p}$. This algebraization of $p$-adic homotopy theory may well be the best possible $p$-adic analogue of Sullivan's rational homotopy theory.

There are many other applications arising from this brave new world of stable homotopy theory, but that is not the subject of this report. Rather I want to talk about brave new worlds, in the plural, and how to travel between them. Several years ago, Jeff Smith initiated a program for an alternative development of the modern foundations of stable homotopy theory based on "symmetric spectra". The full development of that theory lags a few years behind EKMM. Mark Hovey, Brooke Shipley, and Smith [15] are nearly done with a write-up of this version of the stable homotopy category, and they know in general how to develop the homotopy theories of algebras and of commutative algebras in their context, but it may be quite some 
time before that work is written up. Another alternative development, this one based on $\Gamma$-spaces, has been studied by Lydakis and Schwede [22, 33], who build on work of Segal [34], Anderson [2], and Bousfield and Friedlander [6].

In part because EKMM got there first, it has skimmed off the main applications. In fact, there have not yet been many new applications of any alternative approach, and there have not yet been any calculational applications. Moreover, calculations that are based on operad actions and space level $E_{\infty}$-ring structures do not naturally live in the alternative brave new worlds. They do fit naturally into the EKMM setting, since that is an outgrowth of the earlier work which is based on a convenient conceptual repackaging of the relevant operad actions.

Nevertheless, given how central stable homotopy theory is to algebraic topology, it is of real interest to have alternative approaches that involve more elementary and less elaborate structure than is built into the $S$-modules of EKMM. However, it would be willfully perverse to develop such approaches in isolation. The different approaches should give mutually supportive brave new worlds in a single universe, so that one can work in whatever context seems most natural to a particular set of given data. For example, the alternative approaches seem particularly relevant to topological Hochschild homology, which can be developed in any approach, and to topological cyclic homology, whose existing construction naturally involves three different brave new worlds, as I shall briefly indicate later.

Thus what I shall talk about is work in progress of Mike Mandell, Stefan Schwede, Brooke Shipley, and myself $[24,25]$ that shows how to travel between the various brave new worlds of stable homotopy theory. One important feature of our work is its emphasis on the coordinate-free analogue of Smith's coordinatized brave new world. The idea is obvious enough from a comparison of Smith's approach with the concepts introduced by Quinn, Ray, and myself, but it has not previously been taken seriously. This approach may well lead to a pleasant alternative construction of equivariant stable homotopy categories for compact Lie groups. Examples of the kind of equivariant object I have in mind play key roles in the construction of topological cyclic homology of Lars Hesselholt and Ib Madsen [14] and in the proof by John Greenlees and myself [13] of an analogue of the Atiyah-Segal completion theorem that applies to all modules over $M U$.

I will begin by describing a method of constructing symmetric monoidal functor categories wholesale. The basic construction has been well-known to category theorists since the late 1960's (see Day [9]), but Jeff Smith is the first to have seen the relevance of the construction to stable homotopy theory. I will specialize it to 
describe the bottom four categories in the following "Main Diagram":

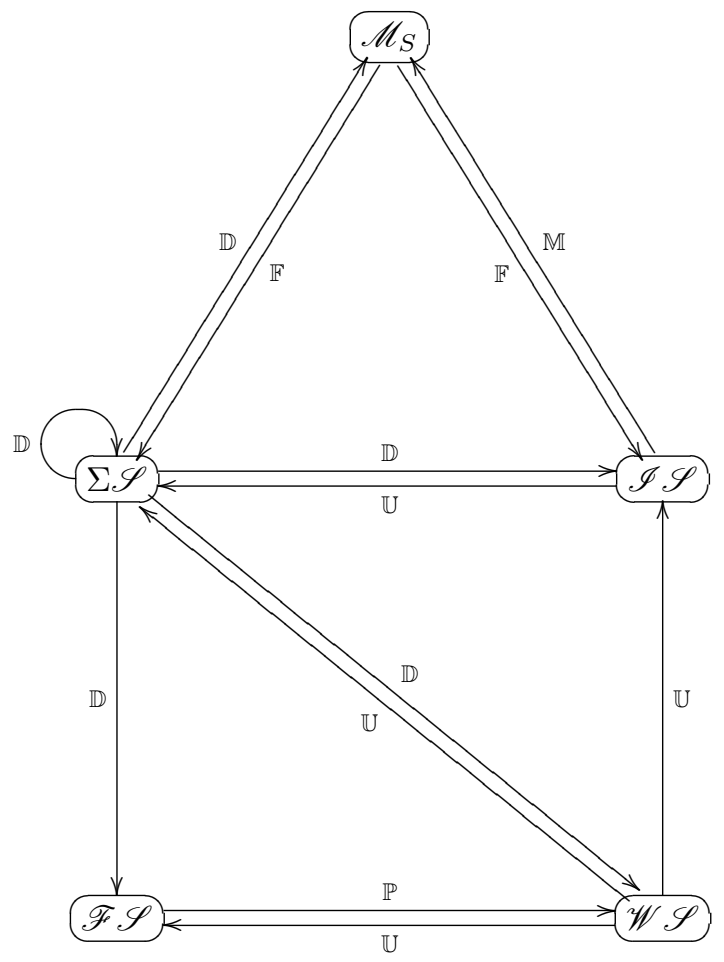

Here $\Sigma \mathscr{S}$ is Smith's category of symmetric spectra, or $\Sigma$-spectra; $\mathscr{I} \mathscr{S}$ is the category of orthogonal spectra, or $\mathscr{I}$-spectra, which is the coordinate-free analogue of Smith's category; $\mathscr{F} \mathscr{S}$ is the category of $\Gamma$-spaces, or $\mathscr{F}$-spectra, whose symmetric monoidal structure was introduced by Lydakis and whose ring objects were studied by Schwede.

I have written $\mathbb{D}$ for all functors defined on $\mathscr{I} \mathscr{S}$ since they are all defined in the same way. We write $\mathbb{D}_{\Sigma}, \mathbb{D}_{\mathscr{I}}, \mathbb{D}_{\mathscr{W}}$, and $\mathbb{D}_{\mathscr{M}_{S}}$ when we wish the notation to record the target category. Similarly, the two functors $\mathbb{F}$ are defined in the same way, and we write $\mathbb{F}_{\Sigma}$ and $\mathbb{F}_{\mathscr{I}}$ when we wish the notation to record the target category.

Roughly speaking, the conclusion of our work is that all approaches are essentially equivalent, so that results proven in one of these categories can be translated into conclusions applicable in another. That is, all composite endofunctors are weakly equivalent to identity functors under appropriate technical hypotheses.

The same conclusions apply to the diagram that is obtained by restricting to monoids in each of these symmetric monoidal categories. That is not quite true of the diagram that is obtained by restricting to commutative monoids. However, there is a notion of an $E_{\infty}$-monoid in each of these categories, and the same conclusions do apply to the diagram that is obtained by restricting to $E_{\infty}$-monoids. In the category of $S$-modules, and in most of the rest of these categories, $E_{\infty}$-monoids are equivalent to commutative monoids.

The general construction of symmetric monoidal functor categories is not limited to applications in stable homotopy theory. For example, as pointed out by Voevodsky, the construction I'm about to give has potential applications to algebraic geometry, where it may shed light on the recent importation of language from 
homotopy theory $[31,38]$. In fact, this may well be the area in which the idea finds its most significant applications.

I will restrict attention to functors that take values in the category of based spaces, but everything can be adapted without difficulty to functors that take values in the category of based simplicial sets. In fact, the basic constructions apply verbatim to functors that take values in any symmetric monoidal category that is tensored and cotensored over the category of based spaces or the category of based simplicial sets.

\section{Symmetric monoidal Categories}

We need to fix some language. A monoidal category $\mathscr{D}$ is a category together with a product $\square=\square_{\mathscr{D}}: \mathscr{D} \times \mathscr{D} \longrightarrow \mathscr{D}$ and a unit object $u=u_{\mathscr{D}}$ such that $\square$ is associative and unital up to coherent natural isomorphism; $\mathscr{D}$ is symmetric monoidal if $\square$ is also commutative up to coherent natural isomorphism.

A functor $F: \mathscr{A} \longrightarrow \mathscr{B}$ between monoidal categories is lax monoidal if there is a map $\lambda: u_{\mathscr{B}} \longrightarrow F\left(u_{\mathscr{A}}\right)$ and a natural map

$$
\phi: F(A) \square_{\mathscr{B}} F(B) \longrightarrow F\left(A \square_{\mathscr{A}} B\right)
$$

such that all coherence diagrams relating the associativity and unit isomorphisms of $\mathscr{A}$ and $\mathscr{B}$ to the maps $\lambda$ and $\phi$ commute. If $\mathscr{A}$ and $\mathscr{B}$ are symmetric monoidal, then $F$ is lax symmetric monoidal if all coherence diagrams relating the associativity, unit, and commutativity isomorphisms of $\mathscr{A}$ and $\mathscr{B}$ commute. The functor $F$ is strict monoidal or symmetric monoidal if $\lambda$ and $\phi$ are isomorphisms.

Lemma 1.1. If $F: \mathscr{A} \longrightarrow \mathscr{B}$ is lax monoidal and $M$ is a monoid in $\mathscr{A}$, then $F(M)$ is a monoid in $\mathscr{B}$. If $F: \mathscr{A} \longrightarrow \mathscr{B}$ is lax symmetric monoidal and $M$ is a commutative monoid in $\mathscr{A}$, then $F(M)$ is a commutative monoid in $\mathscr{B}$.

In the Main Diagram, all categories are symmetric monoidal, all functors except the $\mathbb{D}$ are lax symmetric monoidal, and the $\mathbb{D}$ are lax monoidal. Thus all functors preserve monoids (alias strict ring spectra) and most preserve commutative monoids. The functors $\mathbb{D}$ preserve $E_{\infty}$-monoids, and this gets around the problem that they do not preserve commutative monoids.

\section{2. $\mathscr{D}$-SPACES AND $\mathscr{D}$-SPECTRA}

Let $\mathscr{D}$ be a symmetric monoidal topological category with unit object $u$ and continuous product $\square$. We assume that $\mathscr{D}$ has an initial base object; if not, we implicitly adjoin one. The category $\mathscr{T}$ of based spaces is symmetric monoidal under the smash product with unit object $S^{0}$ and base object a one-point space.

Definition 2.1. A $\mathscr{D}$-space is a based continuous functor $T: \mathscr{D} \longrightarrow \mathscr{T}$. Let $\mathscr{D} \mathscr{T}$ denote the category of $\mathscr{D}$-spaces. For $\mathscr{D}$-spaces $T$ and $T^{\prime}$, define the "external" smash product $T \wedge T^{\prime}$ by

$$
T \wedge T^{\prime}=\wedge \circ\left(T \times T^{\prime}\right): \mathscr{D} \times \mathscr{D} \longrightarrow \mathscr{T}
$$

thus, for objects $d$ and $e$ of $\mathscr{D},\left(T \wedge T^{\prime}\right)(d, e)=T(d) \wedge T^{\prime}(e)$.

Now suppose given a based symmetric monoidal functor $S_{\mathscr{D}}: \mathscr{D} \longrightarrow \mathscr{T}$. We write $S=S_{\mathscr{D}}$ when $\mathscr{D}$ is understood. The theory actually works when $S_{\mathscr{D}}$ is lax symmetric monoidal, but it helps fix ideas to think in terms of the much lesser generality of a strict symmetric monoidal $S_{\mathscr{D}}$. The letter $S$ is meant to suggest 
spheres, since our focus will be on a particular choice of $S$ that is related to the sphere spectrum. However, each $\mathscr{D}$ can have many different choices of $S$.

Definition 2.2. A $\mathscr{D}$-spectrum is a $\mathscr{D}$-space $T: \mathscr{D} \longrightarrow \mathscr{T}$ together with a continuous natural transformation $\sigma: T \bar{\wedge} \longrightarrow T \circ \square$ such that the apppropriate transitivity diagram commutes. Let $\mathscr{D} \mathscr{S}$ denote the category of $\mathscr{D}$-spectra.

We describe the categories $\mathscr{D}$ and functors $S_{\mathscr{D}}$ that we are interested in. We take $S^{n}$ to be the one-point compactification of $\mathbb{R}^{n}$. Similarly, for a finite dimensional real inner product space $V$, we take $S^{V}$ to be the one-point compactification of $V$. Note that the one-point compactification of $\{0\}$ is $S^{0}$.

Example 2.3. Let $\Sigma$ be the (unbased) category of finite sets $\underline{n}=\{1, \ldots, n\}, n \geq 0$, and their permutations; thus there are no maps $\underline{m} \longrightarrow \underline{n}$ for $m \neq n$, and the set of maps $\underline{n} \rightarrow \underline{n}$ is the symmetric group $\Sigma_{n}$. The symmetric monoidal structure is given by concatenation of sets and block sum of permutations, with $\underline{0}$ as unit. The functor $S$ sends $\underline{n}$ to $S^{n}$.

Example 2.4. Let $\mathscr{I}$ be the (unbased) category of finite dimensional real inner product spaces and linear isometric isomorphisms; there are no maps $V \longrightarrow W$ unless $\operatorname{dim} V=\operatorname{dim} W=n$ for some $n \geq 0$, when the space of morphisms $V \longrightarrow W$ is homeomorphic to the orthogonal group $O(n)$. The symmetric monoidal structure is given by direct sums, with $\{0\}$ as unit. The functor $S$ sends $V$ to $S^{V}$. Define a functor $\iota: \Sigma \longrightarrow \mathscr{I}$ by sending $\underline{n}$ to $\mathbb{R}^{n}$ and using the standard inclusions $\Sigma_{n} \longrightarrow O(n)$.

Example 2.5. We can take $\mathscr{D}=\mathscr{T}$. More generally, we can take $\mathscr{D}$ to be any based symmetric monoidal subcategory of $\mathscr{T}$ that contains $S^{0}$. The functor $S_{\mathscr{D}}$ is then the inclusion $\mathscr{D} \longrightarrow \mathscr{T}$. In particular, we let $\mathscr{W}$ be the full subcategory of $\mathscr{T}$ of based spaces homeomorphic to finite $\mathrm{CW}$ complexes. It turns out that $\mathscr{W}$-spaces and $\mathscr{W}$-spectra coincide.

Example 2.6. Let $\mathscr{F}$ be the category of finite based sets $n^{+}=\{0,1, \ldots, n\}$ and based maps, where 0 is the basepoint. This is the opposite of Segal's category $\Gamma$. This category is based with base object $0^{+}$. Take $\square$ to be the smash product of finite based sets; to be precise, we order the non-zero elements of $m^{+} \wedge n^{+}$ lexicographically. The unit object is $1^{+}$. The functor $S$ sends $n^{+}$to $n^{+}$regarded as a discrete based space. Again, it turns out that $\mathscr{F}$-spaces and $\mathscr{F}$-spectra coincide.

\section{FunCtors With SMASH PRODUCT}

Retaining our assumptions on $\mathscr{D}$ and $S=S_{\mathscr{D}}$, we next define $\mathscr{D}$-FSP's. The term "functor with smash product" was introduced by Bökstedt, although examples had appeared earlier. We shall see shortly that $\mathscr{D}$-FSP's give the right notion of a strict

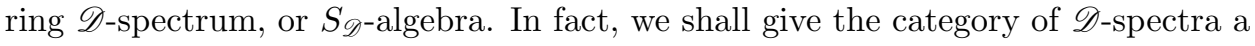
symmetric monoidal structure whose monoids are precisely the $\mathscr{D}$-FSP's.

Definition 3.1. A $\mathscr{D}$-FSP is a $\mathscr{D}$-space $T$ together with a unit map $\eta: S \longrightarrow T$ of $\mathscr{D}$-spaces and a continuous natural product map $\mu: T \bar{\wedge} T^{\prime} \longrightarrow T \circ \square$ such that the appropriate unity, associativity, and centrality of unit diagrams commute. A $\mathscr{D}$-FSP is commutative if the appropriate commutativity diagram commutes.

A $\mathscr{D}$-FSP is a $\mathscr{D}$-spectrum with additional structure. 
Lemma 3.2. A $\mathscr{D}$-FSP has an underlying $\mathscr{D}$-spectrum with structure map

$$
\sigma=\mu \circ(\operatorname{id} \bar{\wedge} \eta): T \bar{\wedge} S \longrightarrow T \circ \square .
$$

Remark 3.3. Up to nomenclature, $\mathscr{D}$-FSP's were first introduced as follows.

(1) A $\mathscr{W}$-FSP is an FSP as introduced by Bökstedt.

(2) A commutative $\mathscr{I}$-FSP is an $\mathscr{I}$-prefunctor as defined by May, Quinn, and Ray. This was the first definition of this sort.

(3) A $\Sigma$-FSP is a symmetric ring spectrum as defined by Smith. Hesselholt and Madsen call these "FSP's defined on spheres".

(4) An $\mathscr{F}$-FSP is a Gamma-ring, as defined by Lydakis and Schwede.

These different kinds of FSP's arise naturally in different applications. $\mathscr{W}$-FSP's are the least general of these examples since restriction along $S_{\mathscr{D}}: \mathscr{D} \longrightarrow \mathscr{W} \subset \mathscr{T}$ shows that a $\mathscr{W}$-FSP is a $\mathscr{D}$-FSP for the other $\mathscr{D}$. Similarly, $\mathscr{I}$-FSP's appear to be less general than $\Sigma$-FSP's since restriction along $\iota: \Sigma \longrightarrow \mathscr{I}$ shows that an $\mathscr{I}$-FSP is a $\Sigma$-FSP. The forgetful functor from $\mathscr{W}$-spaces to $\mathscr{F}$-spaces has a left adjoint "prolongation functor" $\mathbb{P}$ given explicitly by the coends

$$
\mathbb{P} T(X)=\int^{n^{+} \in \mathscr{F}} T\left(n^{+}\right) \wedge X^{n} .
$$

The prolongation of an $\mathscr{F}$-FSP is a $\mathscr{W}$-FSP, and it restricts to give a $\Sigma$-FSP. Of course, these passages from one kind of FSP to another are functorial.

\section{Symmetric monoidal CATEgories of $\mathscr{D}$-SPECTRA}

Now assume that our given symmetric monoidal category $\mathscr{D}$ has a small skeleton $s k \mathscr{D}$. This rules out $\mathscr{T}$ but includes all the really interesting examples. Write $S=S_{\mathscr{D}}$.

Theorem 4.1. The category $\mathscr{D} \mathscr{S}$ of $\mathscr{D}$-spectra has a smash product $\wedge_{S}$ under which it is symmetric monoidal with unit $S$. The categories of $\mathscr{D}$-FSP's and commutative $\mathscr{D}$-FSP's are isomorphic to the categories of monoids in $\mathscr{D} \mathscr{S}$ and of commutative monoids in $\mathscr{D} \mathscr{S}$.

It is natural to introduce alternative names for $\mathscr{D}$-FSP's.

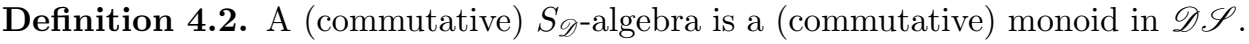

The theorem asserts that these are the same as (commutative) $\mathscr{D}$-FSP's. Thus

$$
\mathscr{D} \text {-FSP's }=\text { monoids in } \mathscr{D} \mathscr{S}=S_{\mathscr{D}} \text {-algebras. }
$$

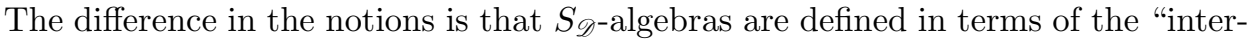
nal" smash product $\wedge_{S}$, whereas $\mathscr{D}$-FSP's are defined equivalently in terms of the more elementary external smash product $\bar{\wedge}$.

Some of the functors between categories of $\mathscr{D}$-FSP's that I described are induced by symmetric monoidal functors between their underlying categories of $\mathscr{D}$-spectra.

Proposition 4.3. The inclusion $\iota: \Sigma \longrightarrow \mathscr{I}$ induces a forgetful functor $\mathbb{U}:$ $\mathscr{I} \mathscr{S} \longrightarrow \Sigma \mathscr{S}$, and $\mathbb{U}$ is lax symmetric monoidal.

Proposition 4.4. Prolongation induces a functor $\mathbb{P}: \mathscr{F} \mathscr{S} \longrightarrow \mathscr{W} \mathscr{S}$, and $\mathbb{P}$ is strict symmetric monoidal. 
To prove the theorem, we first give the category $\mathscr{D} \mathscr{T}$ of $\mathscr{D}$-spaces a smash product $\wedge$ under which it is symmetric monoidal. We do this by internalizing the external smash product $T \bar{\wedge} T^{\prime}$ by taking its left Kan extension along $\square$. The left Kan extension is characterized by the universal property

$$
\mathscr{D} \mathscr{T}\left(T \wedge T^{\prime}, T^{\prime \prime}\right) \cong(\mathscr{D} \times \mathscr{D}) \mathscr{T}\left(T \wedge T^{\prime}, T^{\prime \prime} \circ \square\right)
$$

and it admits two equivalent explicit descriptions.

Definition 4.5. Define the smash product $T \wedge T^{\prime}$ of $\mathscr{D}$-spaces $T$ and $T^{\prime}$ by letting $\left(T \wedge T^{\prime}\right)(d)$ be the space

$$
\left(T \wedge T^{\prime}\right)(d)=\operatorname{colim}_{\phi: e \square f \rightarrow d} T(e) \wedge T^{\prime}(f) .
$$

The colimit is taken over the category $\square / d$ of maps $\phi: e \square f \rightarrow d$. Equivalently, when $\mathscr{D}$ is small, $\left(T \wedge T^{\prime}\right)(d)$ is the coend

$$
\int^{(e, f) \in \mathscr{D} \times \mathscr{D}} \mathscr{D}(e \square f, d) \wedge\left(T(e) \wedge T^{\prime}(f)\right) .
$$

Proposition 4.6. Let $\underline{S}^{0}: \mathscr{D} \longrightarrow \mathscr{T}$ be the functor that sends $d$ to the (based) space $\mathscr{D}(u, d)$. Then $\mathscr{D} \mathscr{T}$ is symmetric monoidal under $\wedge$ with unit object $\underline{S}^{0}$.

The proof is formal. We return to our examples.

Example 4.7. Consider $\mathscr{D}=\Sigma$. The functor $\underline{S}^{0}$ sends $\underline{0}$ to the space $S^{0}$ and sends $\underline{n}$ to a point for $n>0$. For $\Sigma$-spaces $T$ and $T^{\prime}$,

$$
\left(T \wedge T^{\prime}\right)(\underline{n}) \cong \bigvee_{p=0}^{n} \Sigma_{n+} \wedge_{\Sigma_{p} \times \Sigma_{n-p}} T(\underline{p}) \wedge T(\underline{n-p})
$$

as a $\Sigma_{n}$-space.

Example 4.8. Consider $\mathscr{D}=\mathscr{I}$. The functor $\underline{S}^{0}$ sends $\{0\}$ to $S^{0}$ and sends $V$ to a point if $\operatorname{dim} V>0$. For an inner product space $V$ of dimension $n$, choose a subspace $V_{p}$ of dimension $p$ for each $p \leq n$ and let $V-V_{p}$ be the orthogonal complement of $V_{p}$ in $V$. Then, for $\mathscr{I}$-spaces $T$ and $T^{\prime}$,

$$
\left(T \wedge T^{\prime}\right)(V) \cong \bigvee_{p=0}^{n} O(V)+\wedge O\left(V_{p}\right) \times O\left(V-V_{p}\right) T\left(V_{p}\right) \wedge T^{\prime}\left(V-V_{p}\right)
$$

as an $O(V)$-space.

The isomorphisms $S(d) \wedge S(e) \cong S(d \square e)$ specify a natural isomorphism of functors $S \wedge S \cong S \circ \square$. By the universal property, this induces a map of $\mathscr{D}$-spaces $S \wedge S \longrightarrow S$.

Lemma 4.9. The $\mathscr{D}$-space $S_{\mathscr{D}}$ is a commutative monoid in the symmetric monoidal category $\mathscr{D} \mathscr{T}$.

There is an evident notion of a (right) module $T$ over a monoid $S$ in any symmetric monoidal category. The universal property implies the following conceptual description of $\mathscr{D}$-spectra.

Lemma 4.10. The category of $\mathscr{D}$-spectra is isomorphic to the category of $S_{\mathscr{D} \text { - }}$ modules. 
Definition 4.11. For $\mathscr{D}$-spectra $T$ and $T^{\prime}$, thought of as right and left $S$-modules respectively, define $T \wedge_{S} T^{\prime}$ to be the coequalizer in the category of $\mathscr{D}$-spaces (constructed spacewise) displayed in the diagram

$$
T \wedge S \wedge T^{\prime} \frac{\mu \wedge \text { id }}{\text { id } \wedge \mu^{\prime}} \longrightarrow T \wedge T^{\prime} \longrightarrow T \wedge \wedge_{S} T^{\prime},
$$

where $\mu$ and $\mu^{\prime}$ are the given actions of $S$ on $T$ and $T^{\prime}$. Then $T \wedge_{S} T^{\prime}$ inherits a $\mathscr{D}$-spectrum structure from the $\mathscr{D}$-spectrum structure on $T$ or, equivalently, $T^{\prime}$.

Our theorem describing $\mathscr{D}$-FSP's follows formally from the definition and the universal property of left Kan extensions.

Example 4.12. Consider $\mathscr{D}=\mathscr{F}$. Here $S=S_{\mathscr{F}}$ is the unit $\underline{S}^{0}$ of $\wedge$, and an

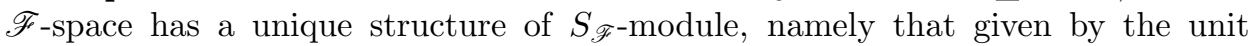
isomorphism $T \wedge S \cong T$. Therefore $\mathscr{F}$-spectra coincide with $\mathscr{F}$-spaces and $\wedge_{S}$ coincides with $\wedge$. The same phenomenon occurs in the case $\mathscr{D}=\mathscr{W}$.

The homotopy theory of $\mathscr{F}$-spectra and $\mathscr{F}$-algebras have been worked out by Bousfield and Friedlander, Lydakis, and Schwede. As I said before, the homotopy theory of $\Sigma$-spectra and $S_{\Sigma}$-algebras is being worked out by Hovey, Smith, and Shipley. The homotopy theory of $\mathscr{I}$-spectra and $S_{\mathscr{I}}$-algebras has not yet been studied. In fact, the desirability of such a study has only become fully apparent with our work. Note that all definitions above apply with $\mathscr{I}$ replaced by $\mathscr{I}_{G}$, the category of finite dimensional orthogonal representations of a compact Lie group $G$ and all linear isometric isometries; this is a topological $G$-category with $G$ acting by conjugation on morphism spaces.

\section{The Definition of $E_{\infty}$ - $\mathscr{D}$-FSP's}

Our comparison results involve the notion of an $E_{\infty}$ - $D$-FSP, which is a $\mathscr{D}$-FSP that is commutative only up to coherence conditions expressed by the action of a certain $E_{\infty}$-operad $\mathscr{E}$.

Let $\mathscr{C}$ be an operad of spaces such that $\mathscr{C}(0)=*$. There is a notion of a $\mathscr{C}$ algebra in $\mathscr{D} \mathscr{S}$ : an action of $\mathscr{C}$ on $T$ is given by $\Sigma_{n}$-equivariant maps of $\mathscr{D}$-spectra

$$
\theta_{n}: \mathscr{C}(n)_{+} \wedge T^{(n)} \longrightarrow T
$$

where $T^{(n)}$ is the $n$th $\wedge_{S}$-power and where the smash product of a space and a $\mathscr{D}$-spectrum is defined in the evident spacewise fashion, $(X \wedge T)(d)=X \wedge T(d)$. The standard associativity, unity, and equivariance diagrams in the definition of an operad action are required to commute. We interpret $T^{(0)}$ to be $S=S_{\mathscr{D}}$, so that $\theta_{0}$ is a unit map $S \longrightarrow T$. This is the more conceptual "internal" form of the definition, but we can translate along the universal property of left Kan extensions to give an "external" form analogous to our original definition of a $\mathscr{D}$-FSP.

There is a canonical $E_{\infty}$-operad $\mathscr{E}$ whose $n$th space is $B \tilde{\Sigma}_{n}$, where $\tilde{G}$ is the translation category of a group $G$. This operad contains the operad $\mathscr{M}$ that defines monoids in any symmetric monoidal category and augments to the operad $\mathscr{N}$ that defines commutative monoids.

Definition 5.2. An $E_{\infty}-\mathscr{D}$-FSP is an $\mathscr{E}$-algebra in $\mathscr{D} \mathscr{S}$.

We have a precisely analogous definition in the context of the $S$-modules of EKMM; I will say a little bit more about what these objects are later, but it is convenient to define $E_{\infty}$-S-algebras now. 
Definition 5.3. An $E_{\infty}$-S-algebra $R$ is an $S$-module together with an action of the operad $\mathscr{E}$ given by maps $\theta: \mathscr{E}(n)_{+} \wedge R^{(n)} \longrightarrow R$ such that the standard associativity, unity, and equivariance diagrams commute. Here $R^{(n)}$ is the $n$th $\wedge_{S}$-power.

In both contexts, it is reasonable to think of $E_{\infty}$-objects as $E_{\infty}$-monoids in the given symmetric monoidal category. $E_{\infty}$-S-algebras must not be confused with $E_{\infty}$-ring spectra, as originally defined in [27]: an $E_{\infty}$-ring structure on a spectrum $E$ is specified in terms of maps

$$
\theta: \mathscr{L}(n) \operatorname{thp} E^{(n)} \longrightarrow E,
$$

where $E^{(n)}$ here is the $n$th external smash product of spectra and thp is the twisted half-smash product. However, the homotopy categories of commutative $S$-algebras, $E_{\infty}$-ring spectra, and $E_{\infty}-S$-algebras are all equivalent, so that only the experts need to worry about the distinction.

\section{The FunCtors $\mathbb{D}$ And $\mathbb{E}$}

All of our categories are now in place, and we can begin to compare them. We first describe functors and natural transformations that compare our categories of $\mathscr{D}$-spectra on a formal level, and we then describe how these comparison maps behave homotopically. Thus we now concentrate on the bottom square of the Main Diagram. We will come back to $S$-modules later.

However, our first construction applies to both $\mathscr{D}$-spectra and $S$-modules. It shows that the category of $\Sigma$-spectra plays a universal role: we can construct an object $\mathbb{D}(T)$ in any of our other categories from a $\Sigma$-spectrum $T$.

Theorem 6.1. Let $\mathscr{C}$ be any symmetric monoidal model category of spectra that is tensored and cotensored over based spaces and has a given sphere object $S$. There is a lax monoidal functor

$$
\mathbb{D}=\mathbb{D}_{\mathscr{C}}: \Sigma \mathscr{S} \longrightarrow \mathscr{C}
$$

Since $\mathbb{D}$ is lax monoidal, it carries $\Sigma$-FSP's to $S$-algebras in $\mathscr{C} ; \mathbb{D}$ is not lax symmetric monoidal, but it carries $E_{\infty}-\Sigma$-FSP's to $E_{\infty}-S$-algebras in $\mathscr{C}$.

The hypotheses on $\mathscr{C}$ in the theorem just mean that it is the kind of modern category of spectra that we have been considering. The construction of $\mathbb{D}$ is based on ideas of Bökstedt [5].

Definition 6.2. Let $I$ be the category of finite sets $\underline{n}=\{1, \ldots, n\}, n \geq 0$, and injective functions.

Definition 6.3. Let $T$ be a $\Sigma$-spectrum. Define

$$
\mathbb{D}(T)=\operatorname{hocolim}_{I} \Omega^{n}(T(\underline{n}) \wedge S) .
$$

Here $\wedge$ is the "tensor" or smash product with spaces in $\mathscr{C}, \Omega^{n}$ is the "cotensor" or function object $F\left(S^{n},-\right)$ in $\mathscr{C}$, and the homotopy colimit is taken in $\mathscr{C}$.

The proof that $\mathbb{D}$ is lax monoidal uses the concatenation functor $\sqcup: I \times I \longrightarrow I$ and formal properties of homotopy colimits, and similarly for the preservation of $E_{\infty}$-structures. However, even when $T$ is a commutative $\Sigma$-FSP, $\mathbb{D}(T)$ is still only an $E_{\infty}$ - algebra in the target category.

Now we give a construction that allows us to compare composites of $\mathbb{D}$ and forgetful functors $\mathbb{U}$ with identity functors. This only works when $\Sigma$ is embedded in $\mathscr{D}$, so it doesn't apply to $\mathscr{F}$. 
Theorem 6.4. For any $\mathscr{D}$ with an embedding $\Sigma \longrightarrow \mathscr{D}$, there is a lax monoidal functor

$$
\mathbb{E}=\mathbb{E}_{\mathscr{D}}: \mathscr{D} \mathscr{S} \longrightarrow \mathscr{D} \mathscr{S}
$$

such that $\mathbb{U E}_{\mathscr{D}}=\mathbb{E}_{\Sigma} \mathbb{U}$. Since $\mathbb{E}$ is lax monoidal, it carries $\mathscr{D}$-FSP's to $\mathscr{D}-F S P$ 's; $\mathbb{E}$ is not lax symmetric monoidal, but it carries $E_{\infty}-\mathscr{D}$-FSP's to $E_{\infty}-\mathscr{D}-F S P$ 's. Moreover, there are monoid and $E_{\infty}$-monoid preserving natural maps

$$
T \stackrel{\alpha}{\longrightarrow} \mathbb{E}(T)<{ }^{\beta} \mathbb{D}_{\mathscr{D}} \mathbb{U}(T)
$$

for $T \in \mathscr{D} \mathscr{S}$.

For a $\mathscr{D}$-spectrum $T$,

$$
\mathbb{E}(T)=\operatorname{hocolim}_{I} \Omega^{n}\left(\Lambda_{n} T\right),
$$

where $\Lambda_{n} T(d)=T(\underline{n} \square d)$. The maps $\alpha$ are given by the inclusions of zeroth terms in homotopy colimits, and the maps $\beta$ are induced by the maps

$$
\Omega^{n} \sigma: \Omega^{n}(T(\underline{n}) \wedge S(d)) \longrightarrow \Omega^{n}(T(\underline{n} \square d)) .
$$

\section{Prespectra, spectra, AND homotopy groups}

We need some standard language to describe the homotopical behavior of $\alpha$ and $\beta$. A coordinatized prespectrum is a sequence of based spaces $T_{n}$ and based maps $\sigma: \Sigma T_{n} \longrightarrow T_{n+1}$. A $\Sigma$-spectrum determines a coordinatized prespectrum by neglect of structure. A coordinate-free prespectrum consists of based spaces $T V$ for finite dimensional sub-inner product spaces of a universe $U \cong \mathbb{R}^{\infty}$, together with a unital and transitive system of based maps $\sigma: \Sigma^{W-V} T V \longrightarrow T(W)$ for $V \subset W$. An $\mathscr{I}$-spectrum determines a coordinate-free prespectrum indexed on any $U$ by neglect of structure. A coordinate-free prespectrum $T$ determines a coordinatized prespectrum with $T_{n}=T\left(\mathbb{R}^{n}\right)$.

A prespectrum of either sort is a spectrum if the adjoints

$$
T_{n} \longrightarrow \Omega T_{n+1} \text { or } T V \longrightarrow \Omega^{W-V} T W
$$

of its structure maps are homeomorphisms. The unmodified term "spectrum" will only be used in this sense. Let $\mathscr{P}$ and $\mathscr{S}$ be the categories of coordinate-free prespectra and spectra indexed on a fixed universe $U$. The suspension spectrum $\Sigma^{\infty} X$ of a based space $X$ has $V$ th space $Q \Sigma^{V} X$, where $Q X=\cup \Omega^{V} \Sigma^{V} X$. We have an adjunction

$$
\mathscr{S}\left(\Sigma^{\infty} X, E\right) \cong \mathscr{T}\left(X, \Omega^{\infty} E\right),
$$

where $\Omega^{\infty}$ is the zeroth space functor. The forgetful functor $\mathscr{S} \longrightarrow \mathscr{P}$ has a left adjoint spectrification functor $L: \mathscr{P} \longrightarrow \mathscr{S}$.

The prespectrum determined by a non-trivial $\mathscr{D}$-spectrum is never a spectrum. Thus $\mathscr{D}$-spectra are never spectra in the sense just defined.

Definition 7.2. The homotopy groups of a coordinatized prespectrum $T=\left\{T_{n}, \sigma_{n}\right\}$ are defined by

$$
\pi_{q}(T)=\operatorname{colim} \pi_{n+q}\left(T_{n}\right) .
$$

A map $f: T \longrightarrow T^{\prime}$ is said to be a $\pi_{*}$-isomorphism if it induces an isomorphism on homotopy groups. More strongly, a map $f: T \longrightarrow T^{\prime}$ of prespectra is a spacewise weak equivalence if each $f: T_{n} \longrightarrow T_{n}^{\prime}$ is a weak homotopy equivalence. A prespectrum $T$ is said to be 
(i) an inclusion prespectrum if each $T_{n} \longrightarrow \Omega T_{n+1}$ is an inclusion.

(ii) convergent if $T_{n} \longrightarrow \Omega T_{n+1}$ is an $(n+\lambda(n))$-equivalence for some nondecreasing sequence $\{\lambda(n)\}$ with infinite limit.

(iii) an $\Omega$-spectrum if each $T_{n} \longrightarrow \Omega T_{n+1}$ is a weak homotopy equivalence.

(iv) an almost $\Omega$-spectrum if each $T_{n} \longrightarrow \Omega T_{n+1}$ for $n>0$ is a weak homotopy equivalence.

These notions have evident reformulations for coordinate-free prespectra, which are equivalent to the notions for the underlying coordinatized prespectrum. We apply these definitions to $\Sigma$-spectra or $\mathscr{I}$-spectra by applying them to their underlying prespectra.

The importance of inclusion prespectra is given by the following fact.

Lemma 7.3. For an inclusion prespectrum $T$, the natural map $\iota: T \longrightarrow L T$ is a $\pi_{*}$-isomorphism.

\section{Homotopical Comparisons between $\Sigma, \mathscr{I}$, AND $\mathscr{F}$-Spectra}

Theorem 8.1. If $T$ is convergent, then $\alpha$ is a $\pi_{*}$-isomorphism and $\beta$ is a spacewise weak equivalence and therefore a $\pi_{*}$-isomorphism.

By the derived category of any of our categories of $\mathscr{D}$-spectra or $\mathscr{D}$-FSP's, we understand the category that is obtained by adjoining formal inverses to the "weak equivalences". Provided that we restrict attention to $\Omega$ - $\mathscr{D}$-spectra, a weak equivalence just means a spacewise weak equivalence when $\mathscr{D}$ is $\Sigma$ or $\mathscr{I}$. I'll come back to what a weak equivalence means more generally later.

Corollary 8.2. The functors

$$
\mathbb{D}_{\mathscr{I}}: \Sigma \mathscr{S} \longrightarrow \mathscr{I} \mathscr{S} \text { and } \mathbb{U}: \mathscr{I} \mathscr{S} \longrightarrow \Sigma \mathscr{S}
$$

induce equivalences between the respective derived categories of $\Omega$-spectra, $\Omega$-FSP's, and $\Omega-E_{\infty} F S P$ 's.

Proof. The required natural spacewise weak equivalences are obtained by restriction from the diagrams

$$
\operatorname{Id}_{\mathscr{I} \mathscr{S}} \stackrel{\alpha}{\longrightarrow} \mathbb{E}_{\mathscr{I}}<\frac{\beta}{\longrightarrow} \mathbb{D}_{\mathscr{I}} \mathbb{U}
$$

and

$$
\operatorname{Id}_{\Sigma \mathscr{S}} \stackrel{\alpha}{\longrightarrow} \mathbb{E}_{\Sigma}<{ }^{\beta} \mathbb{D}_{\Sigma}=\mathbb{U D}_{\mathscr{I}}
$$

We conclude that the more richly structured $\mathscr{I}$-FSP's are really no less general than the $\Sigma$-FSP's.

The methods I have been discussing apply equally well to give a comparison between the categories of $\Sigma$-spectra and of $\mathscr{W}$-spectra. We build $\mathscr{F}$-spectra into the picture by comparing them to $\mathscr{W}$-spectra via homotopical analysis of the prolongation functor. Working simplicially, this analysis has been carried out by Anderson [2], Bousfield and Friedlander [6], and Lydakis [22]. Their conclusions carry over to the topological setting. The prespectra associated to $\mathscr{F}$-spectra are always connective, which gives an intrinsic limitation to this approach. 


\section{The CATEgory of $S$-Modules}

Let $S=\Sigma^{\infty} S^{0}$ be the sphere spectrum. There is a symmetric monoidal category $\mathscr{M}=\mathscr{M}_{S}$ of $S$-modules, where an $S$-module is a spectrum with certain additional

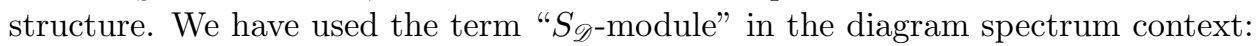
from now on, $S$-modules mean only the kinds of spectra defined in EKMM (=[11]). The smash product in $\mathscr{M}$ will be denoted $\wedge_{S}$. I won't repeat the definitions here. They are based on the idea that prespectra and spectra indexed on inner product spaces should have functoriality in linear isometries built into their structure. Since $S$-modules and $\mathscr{I}$-spectra are both defined in terms of constructions involving naturality on spaces of linear isometries, it is natural to think of the category of $\mathscr{I}$ spectra as a halfway house between the categories of $\Sigma$-spectra and of $S$-modules, partaking of some of the main features of both.

\section{The functor $\mathbb{F}$ from $S$-modules to $\mathscr{D}$-SPECtra}

Returning to the Main Diagram, we describe its functors $\mathbb{F}$. The sphere spectrum $S$ is not $q$-cofibrant in $\mathscr{M}$. This causes the appearance of almost $\Omega$ - $\mathscr{D}$-spectra in the next result.

Theorem 10.1. There are lax symmetric monoidal functors $\mathbb{F}_{\mathscr{D}}: \mathscr{M} \longrightarrow \mathscr{D} \mathscr{S}$, $\mathscr{D}=\Sigma$ and $\mathscr{D}=\mathscr{I}$, such that $\mathbb{F}_{\Sigma}=\mathbb{U} \circ \mathbb{F}_{\mathscr{I}}$. Moreover, these functors take values in the respective subcategories of almost $\Omega$ - $\Sigma$-spectra and almost $\Omega$ - $\mathscr{I}$-spectra.

Before defining the functors, we point out a canonical misconception.

Scholium 10.2. For an $S$-module $M$ and any $\mathscr{D}$, one has a $\mathscr{D}$-space that sends $d$ to $\Omega^{\infty}\left(M \wedge_{S} \Sigma^{\infty} S(d)\right)$. It is a standard mistake to imagine that this gives a $\mathscr{D}$-FSP when $M$ is an $S$-algebra. To obtain a product, one would need that $\left(\Sigma^{\infty}, \Omega^{\infty}\right)$ is an adjoint pair relating $\mathscr{T}$ to $\mathscr{M}$, which is not the case. In fact, as observed by Lewis [19], this cannot be the case for a category as nicely structured as $\mathscr{M}$. This is closely related to the fact that $S$ is not $q$-cofibrant: if it were, we could deduce that its zeroth space $Q S^{0}$ is a commutative monoid, which is clearly false.

The construction that we shall give is a variant of this mistaken idea. Let $S_{S}$ be the canonical $q$-cofibrant approximation of $S$ and let $S_{S}^{(n)}$ be its $n$-fold $\wedge_{S}$-power, with $S_{S}^{(0)}=S$.

Definition 10.3. Let $M$ be an $S$-module. Define

$$
\mathbb{F}_{\Sigma}(M)(\underline{n})=\mathscr{M}\left(S_{S}^{(n)}, M \wedge S^{n}\right) .
$$

Elements of $\Sigma_{n}$ act by conjugation on the specified space of $S$-maps.

The analogue for $\mathscr{I}$ needs a construction. We may view $n \longmapsto S_{S}^{(n)}$ as giving a contravariant strict symmetric monoidal functor $\Sigma \longrightarrow \mathscr{M}$ over the constant functor at $S$, with each map $S_{S}^{(n)} \longrightarrow S$ a weak equivalence.

Lemma 10.4. This functor extends to a continuous contravariant strict symmetric monoidal functor $\mathscr{I} \longrightarrow \mathscr{M}$ over the constant functor at $S$ such that each of its maps $S_{S}^{(V)} \longrightarrow S$ is a weak equivalence.

Definition 10.5. Let $M$ be an $S$-module. Define

$$
\mathbb{F}_{\mathscr{A}}(M)(V)=\mathscr{M}\left(S_{S}^{(V)}, M \wedge S^{V}\right)
$$

Maps of $\mathscr{I}$ act by conjugation on the specified spaces of $S$-maps. 
In both cases, we obtain the $S_{\mathscr{D}}$-module structure required of a $\mathscr{D}$-spectrum by use of the tensor adjunction for the category $\mathscr{M}$ together with smash products of maps. The proof that $\mathbb{F}_{\mathscr{D}}$ is lax symmetric monoidal is formal. It is a basic fact from EKMM that $\mathscr{M}\left(S_{S}, N\right)$ is naturally weakly equivalent to the zeroth space $\Omega^{\infty} N$, and this leads to the proof that the $\mathbb{F}_{\mathscr{D}}(M)$ are almost $\Omega$ - $\mathscr{D}$-spectra.

\section{The functor $\mathbb{M}$ and the composite $\mathbb{M D}$}

I shall say just a little about the proofs of the following results, which finally complete the Main Diagram by describing the functor $\mathbb{M}$.

Theorem 11.1. There is a lax symmetric monoidal functor $\mathbb{M}: \mathscr{I} \mathscr{S} \longrightarrow \mathscr{M}$. If $T$ is an inclusion $\mathscr{I}$-spectrum, then the homotopy groups of $\mathbb{M}(T)$ are naturally isomorphic to the homotopy groups of $T$.

Theorem 11.2. There is a monoidal natural isomorphism $\iota: \mathbb{M D}_{\mathscr{I}} \longrightarrow \mathbb{D}_{\mathscr{M}}$. When applied to an $E_{\infty}-\Sigma-F S P, \iota$ is an isomorphism of $E_{\infty}$-S-algebras.

The functor $\mathbb{M}$ is the composite of three functors:

$$
\mathscr{I} \mathscr{S} \stackrel{P}{\longrightarrow} \mathscr{P}[\mathbb{L}] \stackrel{L}{\longrightarrow} \mathscr{S}[\mathbb{L}] \stackrel{S \wedge \mathscr{L}(-)}{\longrightarrow} \mathscr{M}
$$

The functors $P$ and $L$ are restrictions of the evident functors

$$
\mathscr{I} \mathscr{S} \stackrel{P}{\rightarrow} \mathscr{P} \stackrel{L}{\rightarrow} \mathscr{S} .
$$

Here $P$ takes an $\mathscr{I}$-spectrum to the underlying coordinate-free prespectrum indexed on our given universe $U \cong \mathbb{R}^{\infty}$, and $L$ is the spectrification functor.

The categories $\mathscr{P}[\mathbb{L}]$ and $\mathscr{S}[\mathbb{L}]$ are certain subcategories of the categories of prespectra and spectra. Their objects, called $\mathbb{L}$-prespectra or $\mathbb{L}$-spectra, have extra structure defined in terms of functoriality on linear isometries. The functor $P$ takes values in $\mathscr{P}[\mathbb{L}]$, and the functor $L$ preserves the additional structure. The details were already quite explicit in the early work of Quinn, Ray, and myself, where it was shown that the spectrum associated to a commutative $\mathscr{I}$-FSP is an $E_{\infty}$-ring spectrum. The last functor, $S \wedge \mathscr{L}(-)$, is defined and studied in EKMM; it converts $\mathbb{L}$-spectra to weakly equivalent $S$-modules.

The isomorphism $\mathbb{M D}_{\mathscr{I}} \cong \mathbb{D}_{\mathscr{M}}$ is very illuminating. I promised to say something about what weak equivalences of symmetric spectra are. The definition in terms of localization theory that is used in the formal development of the theory of $\Sigma$-spectra [15] is technical and makes it difficult to identify concretely which maps are weak equivalences. However, Brooke Shipley [35] has given the following curious looking description. We shall ignore a little technicality here.

Theorem 11.3. A map $f$ of $\Sigma$-spectra is a weak equivalence if and only if $\mathbb{D}_{\Sigma} f$ is a $\pi_{*}$-isomorphism.

Obviously $\mathbb{D}_{\Sigma} f$ is a $\pi_{*}$-isomorphism if and only if $\mathbb{D}_{\mathscr{I}} f$ is a $\pi_{*}$-isomorphism. We now see that this is true if and only if $\mathbb{D}_{\mathscr{M}} f$ is a $\pi_{*}$-isomorphism. In $\mathscr{M}$, the weak equivalences are the $\pi_{*}$-isomorphisms.

Corollary 11.4. A map $f$ of $\Sigma$-spectra is a weak equivalence if and only if its associated map of $S$-modules $\mathbb{D}_{\mathscr{M}} f$ is a $\pi_{*}$-isomorphism. 
Thus the definition of a weak equivalence of $\Sigma$-spectra is that dictated by consistency with classical stable homotopy theory: the associated map of $S$-modules, or equivalently spectra, must induce an isomorphism of homotopy groups. This also strongly suggests that the right definition of a weak equivalence of $\mathscr{I}$-spectra is just a $\pi_{*}$-isomorphism, at least on inclusion $\mathscr{I}$-spectra. The restriction corresponds to the technicality ignored in the statement of Shipley's criterion. This suggests that the homotopy theory of $\mathscr{I}$-spectra will work somewhat differently, and perhaps more simply, than the homotopy theory of $\Sigma$-spectra.

I have now described all of the functors in the diagram, but that is only the beginning of our work. The essential point is the comparison of such composites as $\mathbb{F M}: \mathscr{I} \longrightarrow \mathscr{I}$ and $\mathbb{M F}: \mathscr{M}_{S} \longrightarrow \mathscr{M}_{S}$ with identity functors. I will say nothing about that here.

\section{Topological CYClic HOMOLOGY}

Let $T$ be a monoid in any of our symmetric monoidal categories. We can mimic the standard complex for the calculation of Hochschild homology and define $T H H(T)$ to be the geometric realization of the evident simplicial object with $p$-simplices the $(p+1)$ st smash power $T^{(p+1)}$. Since this simplicial object is cyclic, there is an action of the circle group $G$ on the object $T H H(T)$ so constructed. The construction of topological cyclic homology requires a more sophisticated circle action. Although details are work in progress, it seems that the ideas here may lead to significant clarification of the construction.

Certainly Hesselholt and Madsen [14] begin with a $\Sigma$-FSP $T$ and construct from it an "orthogonal $G$-spectrum" ( $\mathscr{I}_{G}$-spectrum). It seems to me that their construction is equivalent to just applying $\mathbb{D}_{\mathscr{I}_{G}}$ to the naive $\mathscr{I}_{G}$-spectrum $T H H(T)$ constructed in the category of $\Sigma$-spectra. This effectively adds in the more sophisticated circle action they require. They then apply the $G$-equivariant version of the functor $\mathbb{M}$ to construct the $G$-spectrum version of $T H H(T)$ from which $T C(T)$ is constructed. The proof that $T H H(T)$ is cyclotomic is really an argument on the $\mathscr{I}_{G}$-spectrum level. Passage to Lewis-May $G$-spectra or, equivalently, EKMM $S_{G}$-modules, then allows the use of the established foundations of equivariant stable homotopy theory. It would be illuminating to have fully developed foundations already on the $\mathscr{I}_{G^{-}}$ spectrum level. There is much work to be done.

\section{REFERENCES}

[1] J.F. Adams. Lectures on generalised cohomology. Springer Lecture Notes in Mathematics Vol. 99, 1969, 1-138.

[2] D. W. Anderson. Chain functos and homology theories. Springer Lecture Notes in Mathematics Vol. 249. 1971, 1-12.

[3] M. Basterra. André-Quillen cohomology of commutative $S$-algebras. Preprint. 1997.

[4] J.M. Boardman. Stable homotopy theory. PhD Thesis, Warwick University, 1964.

[5] M. Bökstedt. Topological Hochschild homology. Preprints, 1985 and later.

[6] A. K. Bousfield and E. M. Friedlander. Homotopy theory of $\Gamma$-spaces, spectra, and bisimplicial sets. Springer Lecture Notes in Mathematics Vol. 658. 1978, 80-130.

[7] D. Burghelea and A. Deleanu. The homotopy category of spectra. I, II, III. Illinois J. Math. 11(1967), 454-473p; Math. Ann. 178(1968), 131-144; Math. Z. 108(1969), 154-170.

[8] F. R. Cohen, T. J. Lada, and J. P May. The Homology of Iterated Loop Spaces. Lecture Notes in Mathematics Vol. 533. Springer-Verlag 1976.

[9] B. Day. On closed categories of functors. Reports of the Midwest Category Seminar IV, Lecture Notes in Mathematics Vol. 137. Springer-Verlag, 1970, pp 1-38.

[10] T. tom Dieck. Bordism of $G$-manifolds and integrality theorems. Topology 9(1970), 345-358. 
[11] A. D. Elmendorf, I. Kriz, M. A. Mandell, and J. P. May. Rings, modules, and algebras in stable homotopy theory. Surveys and Monographs in Mathematics Vol. 47. 1997. American Mathematical Society.

[12] P. Goerss and M.J. Hopkins. In preparation.

[13] J. P. C. Greenlees and J. P. May. Localization and completion theorems for $M U$-module spectra. Annals of Math. To appear.

[14] L. Hesselholt and I. Madsen. On the $K$-theory of finite algebras over Witt vectors of perfect fields. Topology 36(1997), 29-101.

[15] M. Hovey, B. Shipley, and J. Smith. Symmetric spectra. In preparation.

[16] D.M. Kan. Semisimplicial spectra. Illinois J. Math. 7(1963), 463-478.

[17] D.M. Kan and G.W. Whitehead. Orientability and Poincaré duality in general homology theories. Topology 3(1965), 231-270.

[18] I. Kriz. Towers of $E_{\infty}$-ring spectra with an application to $B P$. Preprint. 1995

[19] L. G. Lewis, Jr. Is there a convenient category of spsectra? J. Pure and Applied Algebra 73(1991), 233-246.

[20] L. G. Lewis, Jr., J. P. May, and M. Steinberger (with contributions by J. E. McClure). Equivariant stable homotopy theory. Springer Lecture Notes in Mathematics Vol. 1213. 1986.

[21] E.I. Lima. The Spanier-Whitehead duality in new homotopy categories. Summa Brasil Math. 4(1959), 91-148.

[22] M. Lydakis. Smash-products and Gamma-spaces. Preprint. Bielefeld. 1996.

[23] M. Mandell. $E_{\infty}$ algebras and $p$-adic homotopy theory. PhD Thesis. The University of Chicago. 1997.

[24] M. A. Mandell, J. P. May, S. Schwede and B. Shipley. Diagram spectra and generalized FSP's. In preparation.

[25] M. A. Mandell, J. P. May, S. Schwede and B. Shipley. Diagram spectra and $S$-modules. I. In preparation

[26] J.P. May. Categories of spectra and infinite loop spaces. Lecture Notes in Mathematics Vol. 99, Springer-Verlag 1969, 448-479.

[27] J. P. May (with contributions by F. Quinn, N. Ray, and J. Tornehave). E $E_{\infty}$-ring spaces and $E_{\infty}$-ring spectra. Springer Lecture Notes in Mathematics Vol. 577. 1977.

[28] J.P. May. Infinite loop space theory. Bull. Amer. Math. Soc. 83(1977), 456-494.

[29] J.P. May. Multiplicative infinite loop space theory. J. Pure and Applied Algebra 26(1983), $1-69$.

[30] J. P. May, et al. Equivariant homotopy and cohomology theory. CBMS Regional Conference Series in Mathematics, Number 91. American Mathematical Society. 1996.

[31] F. Morel and V. Voevodsky. Homotopy category of schemes over a base. Preprint. 1996.

[32] D. G. Quillen. On the cohomology and $K$-theory of the general linear groups over a finite field. Annals of Math. (2)96(1972), 552-586.

[33] S. Schwede. Stable homotopical algebra and $\Gamma$-spaces. Preprint, 1997.

[34] G. Segal. Categories and cohomology theories. Topology 13(1974), 293-312.

[35] B. Shipley. Symmetric ring spectra and topological Hochschild homology. Preprint. 1997.

[36] N. Strickland. Products on $M U$-modules. Preprint, 1997.

[37] D. Sullivan. Geometric topology. I. Localization, periodicity, and Galois symmetry. MIT. Mimeographed notes. Circa 1972.

[38] V. Voevodsky. The Milnor conjecture. Preprint. 1996.

[39] J. Wolbert. Classifying modules over $K$-theory spectra. J. Pure and Applied Algebra. To appear.

The University of Chicago, Chicago, IL 60637

E-mail address: may@@math.uchicago.edu 